\title{
Biomarkers in peripheral blood detect acute rejection
}

Diagnosis of acute renal allograft rejection (AR) currently requires confirmation by renal biopsy-an invasive process that is limited by sampling error and assessment variability. Findings from a new study, however, suggest that AR could instead be diagnosed by assessing biomarkers present in peripheral blood, potentially avoiding the need for costly and invasive renal biopsies. "This test could change metrics for patient care and provide the

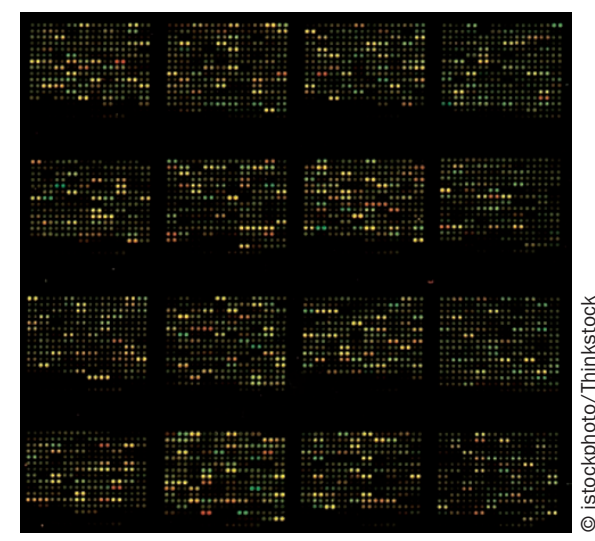

first non-invasive read out of immune injury and safety", says researcher Minnie Sarwal.

To develop a test for the sensitive and specific diagnosis of acute rejection in peripheral blood, Li et al. used microarrays and quantitative PCR to identify genes that distinguished patients with AR from those with either no evidence of rejection or with other clinical phenotypes. The study used a total of 367 blood samples, each of which was matched with a protocol biopsy sample.

Using three different microarray platforms, the researchers first identified 2,382 genes that were differentially expressed in peripheral blood from 60 patients with biopsy-proven AR and 62 patients without rejection, from a single centre. They then used quantitative PCR to verify the differential expression of a subset of genes, and applied a logistic regression model to identify a set of five genes (DUSP1, PBEF1, PSEN1,
MAPK9, and NKTR) that identified AR in a separate verification cohort $(n=47)$. The five-gene model was independently validated in 198 blood samples collected from 12 different transplant centres as part of an NIH-funded prospective randomized trial, where all data analysis was independently performed and validated by biostatisticians at the NIH. The gene set distinguished AR from no rejection with $91 \%$ sensitivity and $94 \%$ specificity and from other pathologies with $91 \%$ sensitivity and $90 \%$ specificity. "This blood test has excellent negative predictive value for biopsy-proven $\mathrm{AR}$, which means that patients can be serially assessed for immunological safety and unnecessary protocol biopsies can be avoided", concludes Sarwal.

Susan J. Allison

Original article Li, L. et al. A Peripheral blood diagnostic test for acute rejection in renal transplantation. Am. J. Transplant. doi:10.1111/j.1600-6143.2012.04253.x 